\title{
BER Performance in Wavelength Packet Switched WDM systems \\ during Nano-second Wavelength Switching Events
}

\author{
A. Dantcha ${ }^{1}$, L.P. Barry ${ }^{1}$, J. Murphy ${ }^{1}$, J.Dunne ${ }^{2}$ T. Mullane ${ }^{2}$ and D. McDonald ${ }^{2}$ \\ (1) Research Institute for Network and Communications Engineering, \\ School of Electronic Engineering, DCU, Dublin 9, IRELAND. Email : liam.barry@dcu.ie \\ (2) Intune Technologies Ltd, 9c Beckett Way, Park West, Dublin 12, IRELAND
}

\begin{abstract}
An important characteristic of wavelength tuneable laser transmitters is that as they tune between output wavelength channels they may generate light at a range of other wavelengths. This effect may ultimately influence the design of WDM wavelength packetswitched networks employing wavelength tuneable transmitters. We have investigated this effect by examining the BER transmission performance of a WDM channel as a function of the degree of attenuation of another WDM wavelength signal during fast wavelength switching events. Our results show the importance of attenuating the output signal from the wavelength switched laser in order to prevent performance degradations on the monitored data channel.
\end{abstract}

Indexing Keywords: Optical communications, networks, wavelength division multiplexing, tuneable laser, packet switching, cross-channel interference. 


\section{Introduction}

Wavelength tuneable lasers are rapidly becoming a mainstream component in photonic networks. In addition to providing immediate cost saving for Wavelength Division Multiplexed (WDM) networks in the areas of back-up transmitters and inventory reduction, these tuneable devices may also be used for developing new network architectures in which wavelength channels can be provided where and when needed. One solution for extremely efficient bandwidth utilisation in WDM networks is the use of wavelength packet routing [14], in which the WDM optical packets are generated by a fast tuneable light source in conjunction with an external modulator. The wavelength packets can then be routed to specific nodes in the optical network by using simple optical filtering techniques. The use of wavelength packet transmission is also a cost-effective path for the migration from single wavelength SDH/SONET based networks to full WDM systems (which can involve a costly introduction of new equipment for each WDM channel installed).

In a complete WDM system that employs wavelength packet switching, each transmitter can tune its emission wavelength to transmit packets of information at a number of different wavelengths. The information from all the transmitters can then be multiplexed together and sent over optical fibre. The composite wavelength signal may then pass through an Array Waveguide Grating (AWG), which sends each of the incoming wavelengths to one specific output fibre port. In general it is possible to write the wavelength connectivity in the form of a matrix. Thus by choosing an appropriate wavelength on the transmitter side, the laser selects the output port to which the information packet is sent. The tuneable transmitters, together with the optical coupler and the AWG, become a strictly non-blocking switch fabric with a switching speed equal to the tuning speed of the laser $[5,6]$. 
Clearly the key component for implementing such networks is the wavelength tuneable laser transmitter, as the overall design of the wavelength packet-switched WDM networks will be heavily dependent on the characteristics of these tuneable devices. The main characteristics that determine the usefulness of wavelength tuneable lasers in wavelength packet-switched systems are the wavelength tuning range, the side-mode suppression ratio (SMSR), the output power, and the speed at which the device can switch from one wavelength to another. Taking into account these characteristics, the most suitable tuneable lasers for use in wavelength packet-switched systems are electronically tuneable devices [7]. Typical examples of such devices are grating-assisted co-directional coupler with sampling grating reflector (GCSR) lasers [3,8], the Sample Grating distributed Bragg reflector structure [9] and super structure grating distributed Bragg reflector (SSG-DBR) lasers [10-12]. These devices can achieve tuning ranges in excess of $60 \mathrm{~nm}$, SMSR's exceeding $40 \mathrm{~dB}$, output powers above $10 \mathrm{dBm}$, and switching times in the order or 20-50 ns. Another important feature of these electronically tuneable lasers is that as the transmitter is being switched between a low and a high wavelength channel, it is possible that the device will emit intermediate wavelengths during the switching event. This effect may clearly cause serious problems in an overall wavelength packet-switched WDM network as the optical output from the tuneable laser, as it transitions between two specific wavelengths, may result in cross-channel interference with another wavelength channels in the WDM network. This may ultimately limit the Bit-Error-Rate (BER) performance of specific data channels in the network. 
The work carried out in this paper was undertaken using a nano-second wavelength switched laser module that uses DBR technology. We have initially characterised the switching time of this module by encoding a data packet onto the tuneable laser output, such that the data spans the transition period between two output wavelengths, and then examining the eye diagram of the resulting signal at both output wavelengths. From this work we have determined the switching time (during which we are unable to successfully encode data onto the laser output) to be around $38 \mathrm{~ns}$. We have then multiplexed the output from the tuneable laser (TL) module with a second laser transmitter (that is generating data at a wavelength channel that lies between the 2 wavelengths that the TL is switching between). From this work the degradation in system performance on the central data channel, due to the spurious wavelength signals emitted from the TL during the switching event, is confirmed by the presence of an error floor in the BER vs. received power measurements. We proceed to examine the levels of attenuation required at the output of the TL module such we can obtain BER's of $10^{-9}$ or better for the data channel that lies between the two wavelengths that the TL module is switching between.

\section{Determination of tunable laser switching time}

To determine the switching time of the TL module we have employed the experimental testbed as presented in Fig.1. The module is controlled via a PC and can be set to transition between different wavelengths on the ITU grid. A trigger output from the wavelength tunable module is available to determine the timing of the transition between wavelengths from the TL. This trigger signal is then fed into a signal generator that can produce variable width pulse bursts at the same repetition rate that the laser transitions from one wavelength to another. The output from this signal generator is subsequently fed into the Pattern 
Generator such that bursts of data, at a rate of $2.5 \mathrm{Gbit} / \mathrm{s}$, can be applied to one specific wavelength channel, and the length of the burst and position of the burst relative to the laser transition time, can be varied using the signal generator.

The information is encoded onto the optical output from the TL using an external modulator with a bandwidth $8 \mathrm{GHz}$. The data signal from the pattern generator is fed directly into the RF port of the modulator while the bias port is connected to a voltage supply (this is optimized during the experiments to achieve the clearest eye opening). The optical data signal from the modulator is then amplified before an optical filter (bandwidth: $0.28 \mathrm{~nm}$ ) is used to select out one specific wavelength channel. The optical data signal is then detected using a $50 \mathrm{GHz}$ pin diode and displayed on an oscilloscope.

The TL module is initially set to transition back and forth between $1533 \mathrm{~nm}$ and $1538 \mathrm{~nm}$ at a repetition rate of $50 \mathrm{kHz}$. Data bursts from the pattern generator are applied to the optical output from the tuneable laser as described above. By varying the relative delay between the time when the data burst is applied and the time when the TL transitions between its two output channels, the information burst can be put on one of the two wavelengths, or set to sit across the transition between the two wavelengths. By triggering the oscilloscope on the burst signal we can view the data packets being generated at each wavelength. The position of the data packet applied to the modulator was set so as to span the transition from 1533 to $1538 \mathrm{~nm}$, and we examined the data output on both of the wavelength channels by tuning the optical filter between the two wavelengths. For the output at $1533 \mathrm{~nm}$ the initial bits of the data packet are clean, but as we approach the transition, and the output wavelength moves away from $1533 \mathrm{~nm}$, the eye starts to close (as shown in Fig. 2). The time interval for which 
the eye diagram is completely open and the TL can be used for error-free transmission at this wavelength is measured to be $248 \mathrm{~ns}$. We then tuned the filter to select the output at 1538 $\mathrm{nm}$, in this case the final bits of the data packet are clean, but the initial bits have closed eyes due to the fact the laser is in the process of transitioning to $1538 \mathrm{~nm}$ from $1533 \mathrm{~nm}$ (Fig. 3). The time interval for which the bits are clean and the TL can be used for error-free transmission at this wavelength is $202 \mathrm{~ns}$. By adding the time intervals for which the TL module can be used for error-free transmission at the two wavelengths it is transitioning between, we obtain a total of $449 \mathrm{~ns}$. As the total length of the data bust is $487 \mathrm{~ns}$, we can determine that the transition time (switching time) during which we are unable to transmit data error-free on either of the output wavelengths is $38 \mathrm{~ns}$. This switching time has been verified by direct measurement of the wavelength switching event itself, using a time resolved spectral measurement technique.

\section{Effects of TL output during switching event}

To investigate how the BER transmission performance of a WDM channel is affected by the output from the TL module that is transitioning between two wavelengths on either side of the data channel being monitored, we have used the experimental set-up shown in Fig. 4. The set-up is similar to that used in Fig. 1, but in addition to the TL we use a HP wavelength tunable external cavity laser (ECL) that can emit light from 1480 to $1570 \mathrm{~nm}$. $2.5 \mathrm{Gbit} / \mathrm{s}$ electrical data signal $\left(2^{11}-1\right.$ PRBS $)$ from the pattern generator is encoded onto the optical signal from the ECL laser using the external modulator, and this data signal is then coupled together with the output from the TL module. The data channel from the ECL can be set to any wavelength between the two output wavelengths from the TL module. A characteristic of this module is that as it transitions between two specific channels it may excite other 
wavelength channels that are being used for data transfer in an overall WDM network. The purpose of this set-up is to determine how this affect's the performance of information transfer in a WDM network. Fig. 5 displays the composite wavelength signal after the data channel from the ECL (which has been set to $1535.4 \mathrm{~nm}$ ) is combined with the TL output, with the power levels in the three wavelength signals having been equalized. The composite signal then passes through an optical filter, with a bandwidth of $0.28 \mathrm{~nm}$ that selects the $1535.4 \mathrm{~nm}$ data channel. The optical data signal is then detected and displayed on an oscilloscope, or input into the error analyzer to determine the BER of the received signal.

To determine whether the signals excited by the tunable module (as it transitions between two wavelengths on either side of the data channel) affect the system performance, we must first of all plot the back-to-back performance of the $1535.4 \mathrm{~nm}$ data channel. The BER vs. received power for the back-to-back case is shown in Fig. 6. It should be noted that the low receiver sensitivities in this figure are as a result of the low receiver gain used in the present experiment. We then proceed to measure the BER vs. received power for the case when the data channel is coupled together with the TL module output before being filtered out (also shown in Fig. 6, $0 \mathrm{~dB}$ attenuation curve). The associated eye diagrams of the received data signals for the single channel case, and the case when the data channel is multiplexed with the tunable laser (that is switching between two wavelengths), before being filtered out and detected, are shown in Fig. 7. From these eye diagrams we can clearly see the noise added to the data signal as a result of the TL laser generating a spurious wavelength output at the same wavelength as the monitored data channel, during its transition between two output wavelengths. The effect of this on the BER vs. received power curve is to place an error floor (at $4 \times 10^{-5}$ as shown in Fig. 6) on the performance of the monitored data channel. 
Similar error floors were obtained when the data channel was set to wavelengths corresponding to the other spurious wavelengths that are generated during the transition period of the TL module. Fig. 8 displays the BER vs. received power curves for the case when the ECL is tuned to three different wavelength channels (1534.2, 1535.4, and 1536.6 $\mathrm{nm}$ ) between the output wavelengths being emitted from the TL.

From the measured error-floor it is possible to estimate the length of time that the TL output is at the same wavelength as the monitored data channel, during its transition. This can be achieved in the following manner: the TL is transitioning between its two output wavelengths every 10 microseconds, and since we are sending information at $2.5 \mathrm{Gbit} / \mathrm{s}$, in 10 microseconds we send approx. 25000 bits. With a BER of $4 \times 10^{-5}$, this means that 1 of the 25000 bits sent in 10 microseconds is received in error due to the excitation of the intermediary wavelength channel. However given that the signal generation from the tunable laser only gives an error for a sent "0", and given that we send unbiased data (equal number of " 1 's" and " 0 's"), we can assume that the intermediary wavelength is on for approx. 2 bit periods of the $2.5 \mathrm{Gbit} / \mathrm{s}$ data signal, or $800 \mathrm{ps}$.

The limitation on BER performance on the monitored data channel that we have investigated in this work (due to the spurious wavelength output from the TL module during it's transition period) is clearly a major issue for the development of large-scale WDM networks employing tunable lasers for wavelength packet-switched architectures. In order to overcome these limitations in real WDM communication systems using wavelength packet switching, it will be vital to extinguish the output from the TL as it transitions between specific wavelength channels. This will ensure that the spurious wavelengths emitted from the 
tunable laser as it transitions, do not limit system performance. To determine the required level of signal extinction at the TL module output, such that it will not affect system performance in a wavelength packet-switched WDM system, we have used the experimental arrangement described earlier. In this work we have examined how the BER floor on the filtered data channel, varies as the output of the TL module is attenuated. These results are shown in Fig. 6 where we can see that it is necessary to attenuate the output of the tunable laser module by $8 \mathrm{~dB}$ to ensure that the BER of the received signal is kept above $10^{-9}$. This result is for the case of one data channel being degraded by a single switching event (i.e. one tunable laser switching back and forth across the data channel). The TL attenuation requirements for the case when there is simultaneous switching of multiple TL modules, in a WDM packet switched system, will clearly exceed $8 \mathrm{~dB}$ in order to achieve error free performance on all wavelength channels. This area is currently under investigation.

\section{Conclusions}

Wavelength tunable lasers are becoming a mainstream component in photonic networks. In addition to providing cost saving for WDM networks with respect to inventory reduction, these tunable devices may also be used for implementing more efficient bandwidth utilization in WDM networks by employing wavelength packet switching architectures. In these networks, WDM optical packets are generated using fast tunable light sources, and then routed to specific nodes in the optical network by using optical filtering techniques. An important characteristic of electrically tunable laser devices, that may influence their operation in WDM packet-switched systems, is that as they transition between two output wavelengths they may generate light at intermediary wavelengths. This will clearly affect the performance of other data channels in the system that are transmitting on the same 
wavelengths as those generated by a tunable transmitter during a switching event. In this work we have shown how this effect limits the performance of a data channel by placing an error floor on the BER vs. received power characteristic of that channel. To overcome these performance limitations, care must be taken to ensure adequate attenuation of the output of a laser transmitter under high speed switching operation. This will ensure that BER performance degradation due to the interference of wavelength signals produced during switching can be controlled. Ideally, the laser transmitter itself should perform the attenuation function and advances in integration technology are making this a cost-effective approach [2].

\section{References}

1. K. Shrikhande et al., "Performance demonstration of fast-tuneable transmitter and burst-mode packet receiver for HORNET" Optical Fibre Communication Conference 2001, Technical Digest, paper ThG2-1, Annaheim, USA, 2001.

2. I. White, R. Plenty, M. Webster, Y.J. Chai, and A. Wonfor, "Wavelength switching components for future photonic networks”, IEEE Communications Magazine, vol. 40, pp. $74-81,2002$.

3. Chun-Kit Chan, K. Sherman, and M. Zirngibl, “ A fast 100 channel wavelength tunable transmitter for optical packet switching”, IEEE Photonics Technology Letters, vol. 13, pp 729 - 731, July 2001.

4. M. Duser, I. De Miguel, P. Bayvel, D. Wischik, "Timescale analysis for wavelengthrouted optical burst-switched (WR-OBS) networks", Optical Fibre Communication Conference 2001, Technical Digest, paper WG7, Annaheim, USA, 2001 
5. D. Sadot, and I. Elhanany, "Optical switching speed requirements for terabit/second packet over WDM networks", IEEE Photonics Technology Letters, vol. 12, pp. 440 $442,2000$.

6. S.M. Gemelos, I.M. White,D. Wonglumsom, K. Shrikhande, T. One,L.G. Kazovsky, "WDM metropolitan area network based on CSMA/CA packet switching", IEEE Photonics Technology Letters, Vol. 11, pp. 1512-1514, 1999.

7. Larry Coldren, "Monolithic Tunable Diode Lasers", IEEE Journal of Selected Topics in Quantum Electronics, vol.6, pp. 989-999, 2000.

8. P.J. Rigole, S. Nilsson, L. Backbom, B. Stalnacke, E. Berglind, J.P. Weber, and B. Stoltz, "Quasi-continuous tuning range from 1560 to $1520 \mathrm{~nm}$ in a GCSR laser with high power and low tuning currents", IEE Electron. Lett., vol. 32, pp. 2352 - 2354, 1996.

9. B Mason, "Characteristics of sampled grating DBR lasers with Integrated Optical Amplifiers", Optical Fibre Communication Conference 2001, Technical Digest, paper Tul6, Annaheim, USA.

10. H. Ishii, H. Tanobe, F. Kano, Y. Tohmori, Y. Kondo, and Y. Yoshikuni, "Quaiscontinuous wavelength tuning in super-structure grating (SSG) DBR lasers", IEEE J. Quantum Electron., vol. 32, pp. 433-440, 1996.

11. G. Sarlet, G. Morthier, R. Baets, "Wavelength and mode stabilization of widely tunable SG-DBR and SSG-DBR lasers", IEEE Photonics Technology Letters, Vol. 11, pp. 1351-1353, 1999.

12. G. Sarlet, G. Morthier, R. Baets, "Control of widely tunable SSG-DBR lasers for dense wavelength division multiplexing", J. Lightwave Technology, Vol. 18, pp. 1128-1138, 2000. 



\section{Figure Captions}

Fig. 1 Experimental configuration to determine switching time of TL module.

Fig. 2 Portion of received data packet at $1533 \mathrm{~nm}$ with data encoded onto TL module such that it spans transition from 1533 to $1538 \mathrm{~nm}$.

Fig. 3 Portion of received data packet at $1538 \mathrm{~nm}$ with data encoded onto TL module such that it spans transition from 1533 to $1538 \mathrm{~nm}$.

Fig. 4 Experimental configuration to determine how the spurious wavelength signals emitted during switching of TL module affect multiplexed data channel lying between output wavelengths from TL.

Fig. 5 Composite wavelength signal after coupling together output from TL module (switching back and forth between 1533 and $1538 \mathrm{~nm}$ ) and data channel from ECL at $1535.5 \mathrm{~nm}$.

Fig. 6 BER vs. received power for back-to-back case, and for case when the data channel is multiplexed with output from the TL Module (as a function of attenuation of the TL output).

Fig. 7 Received eye diagrams for the $1535.5 \mathrm{~nm}$ channel for (a) the back-to-back case, and (b) for the case when the data channel is multiplexed with the tunable laser output before being filtered out and detected.

Fig. 8 BER vs. received power when the data channel from ECL is tuned to three different wavelengths between output wavelengths from TL module (triangles : $1534.2 \mathrm{~nm}$; squares : $1535.4 \mathrm{~nm}$; circles : $1536.6 \mathrm{~nm})$. 


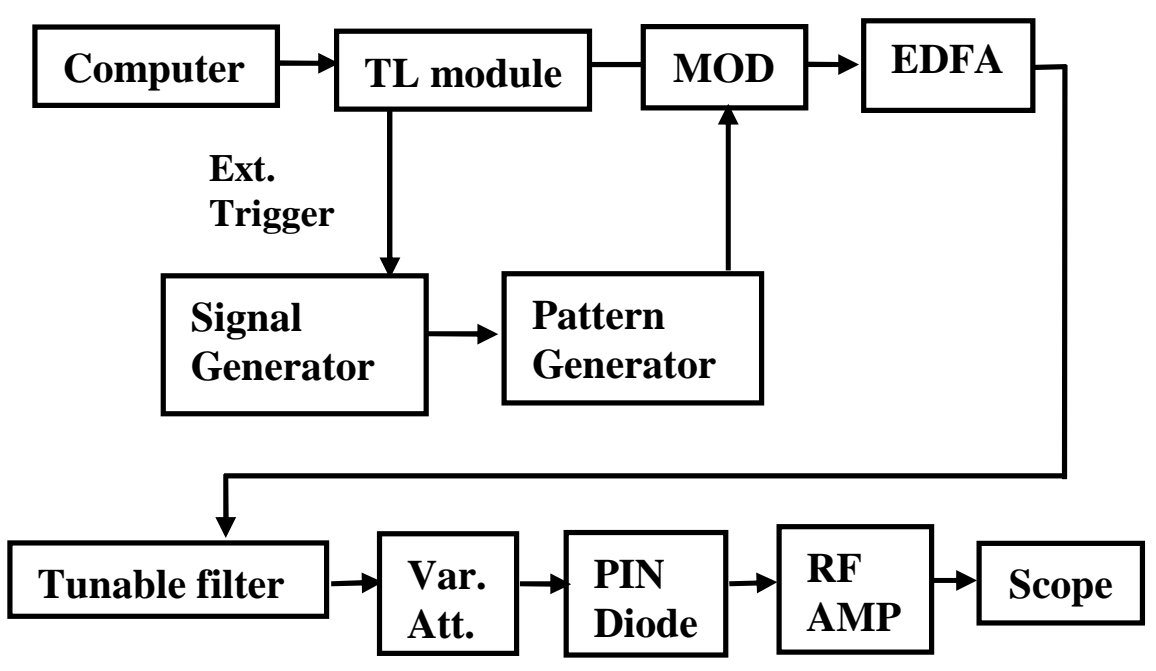

FIGURE 1

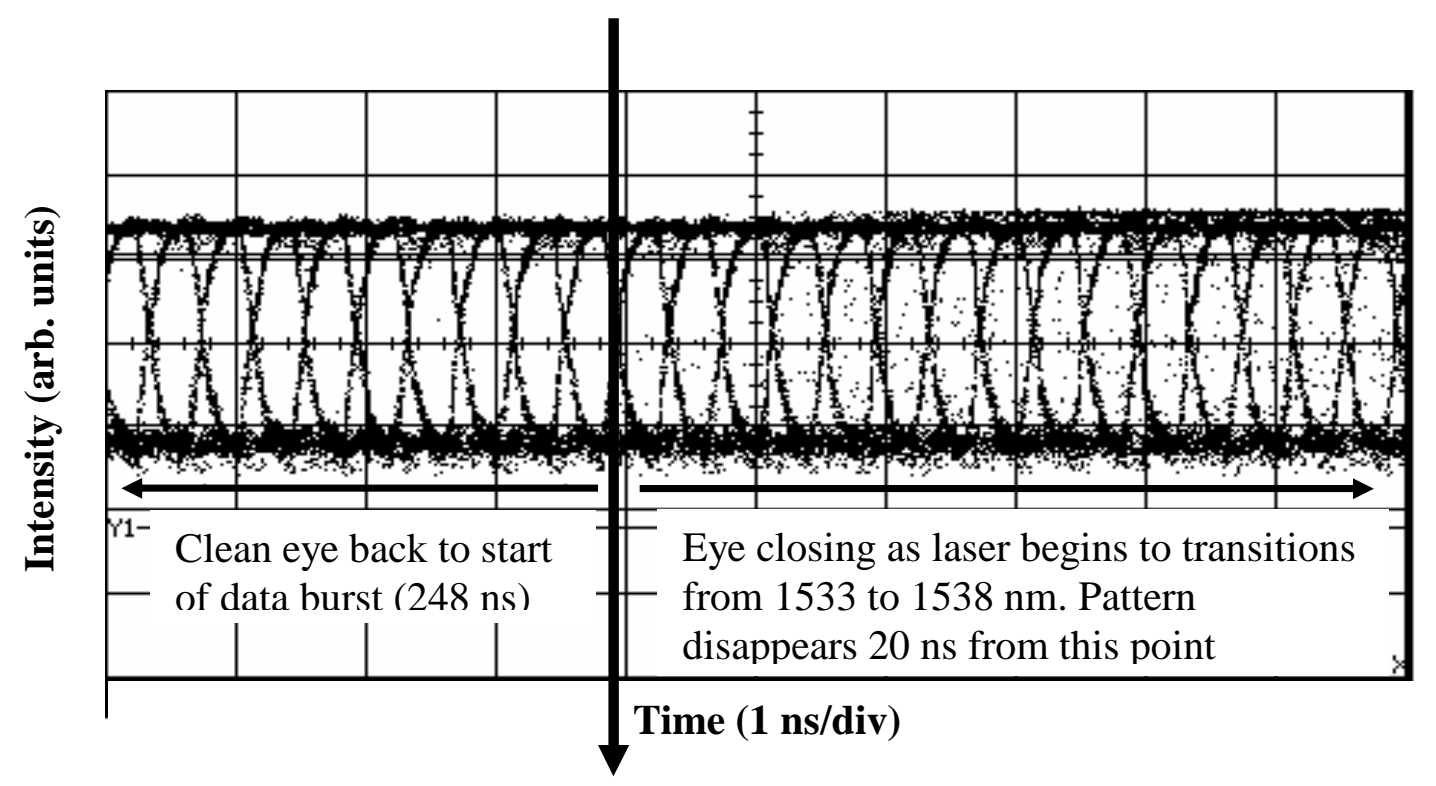

FIGURE 2 


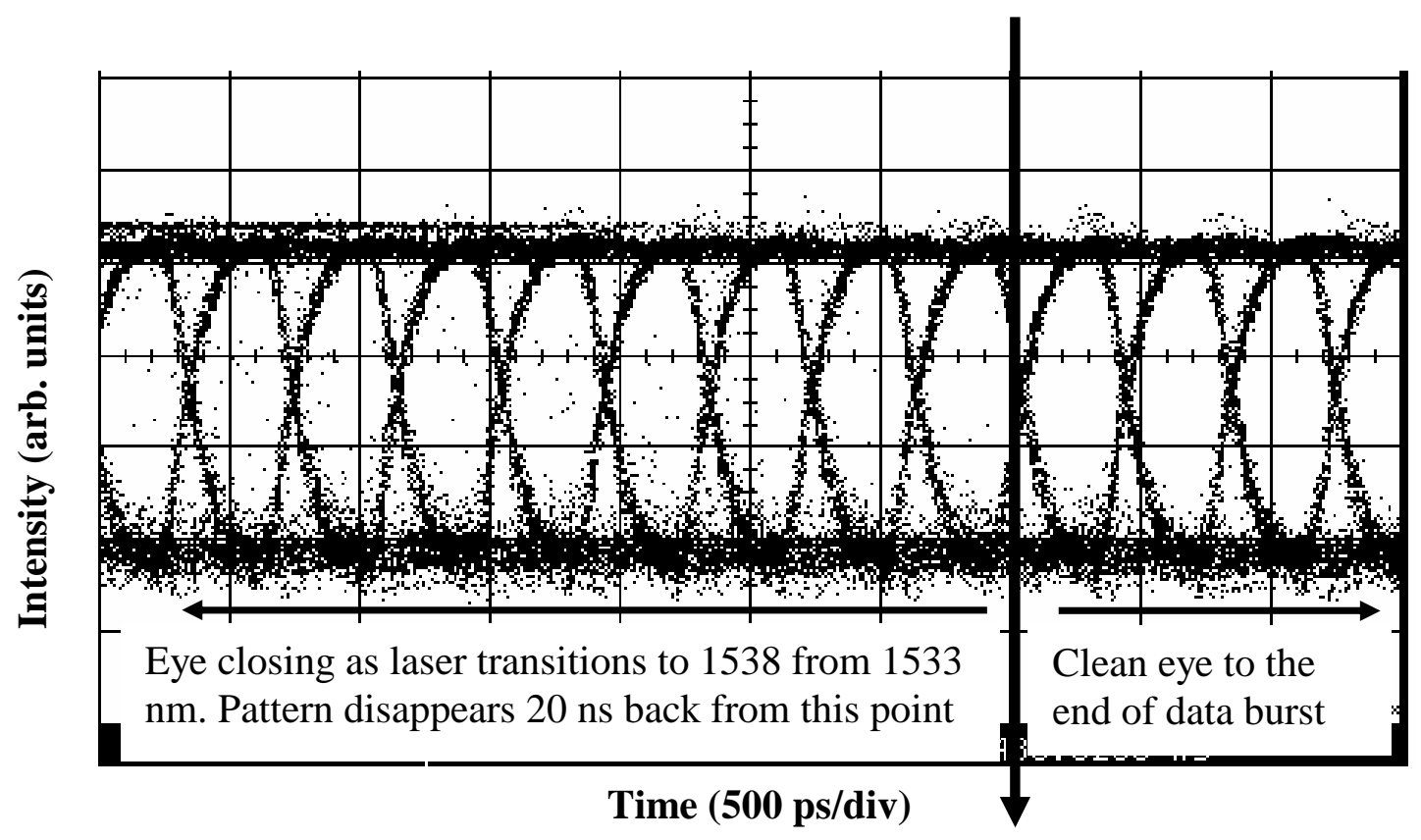

FIGURE 3

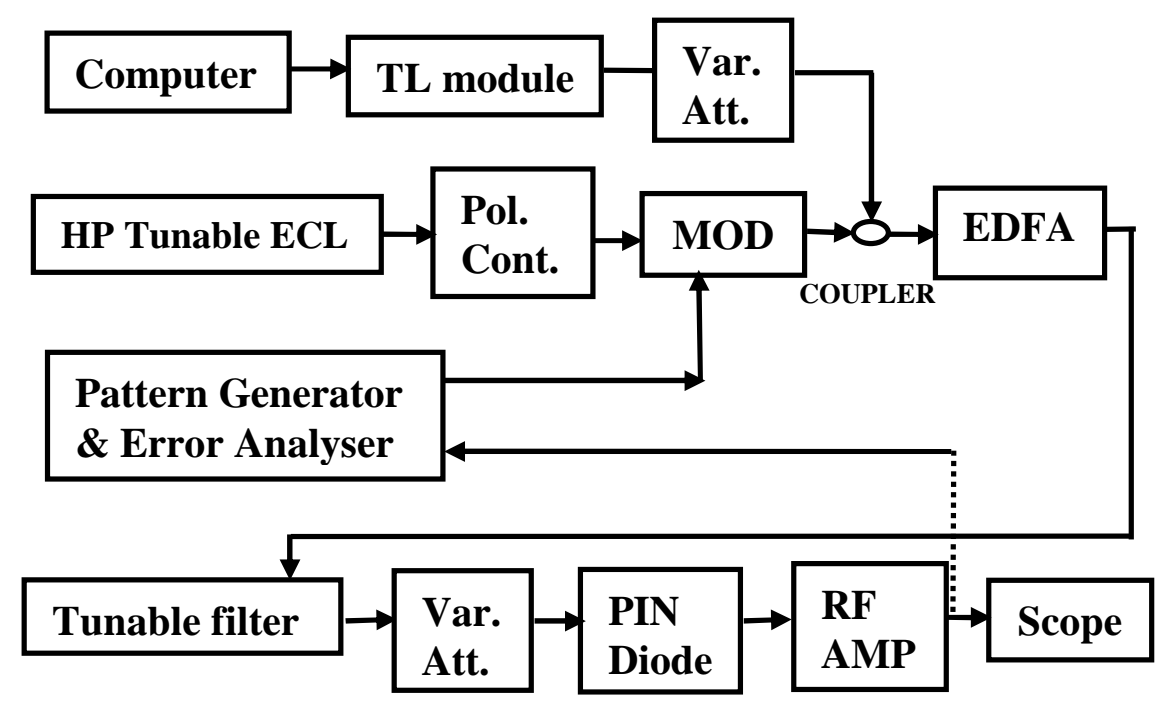

FIGURE 4 


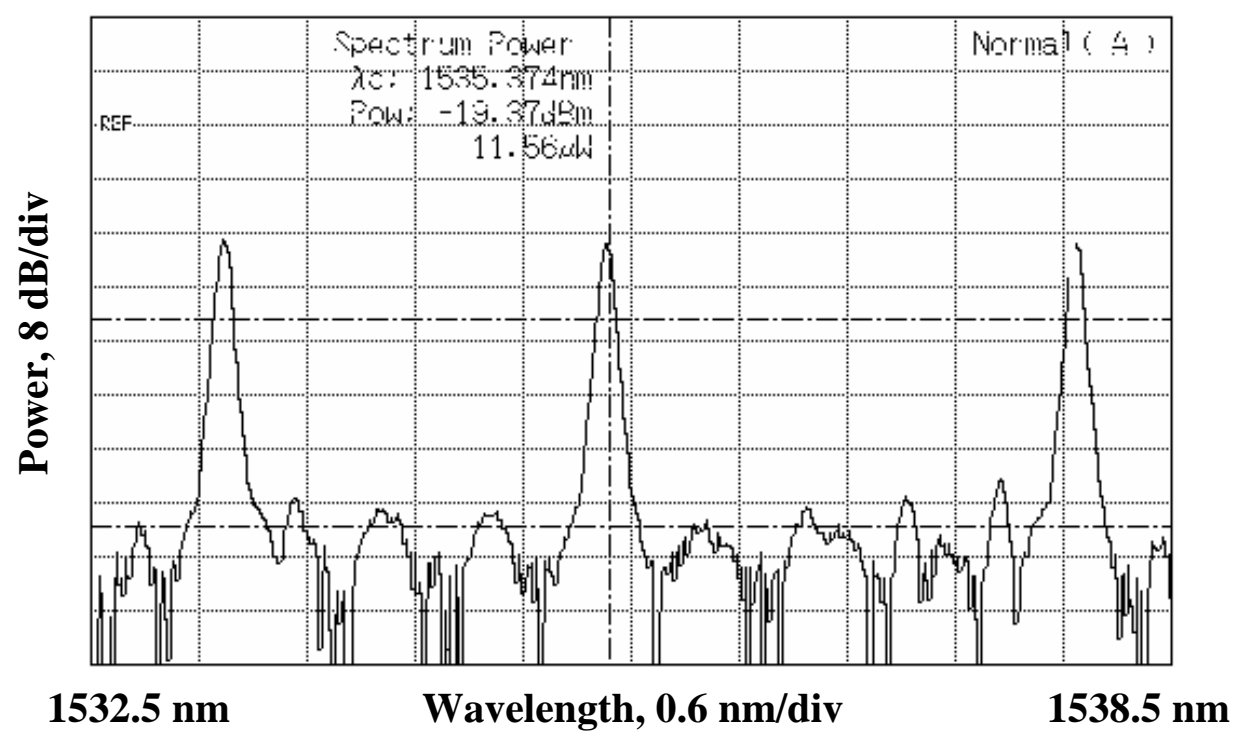

\section{FIGURE 5}

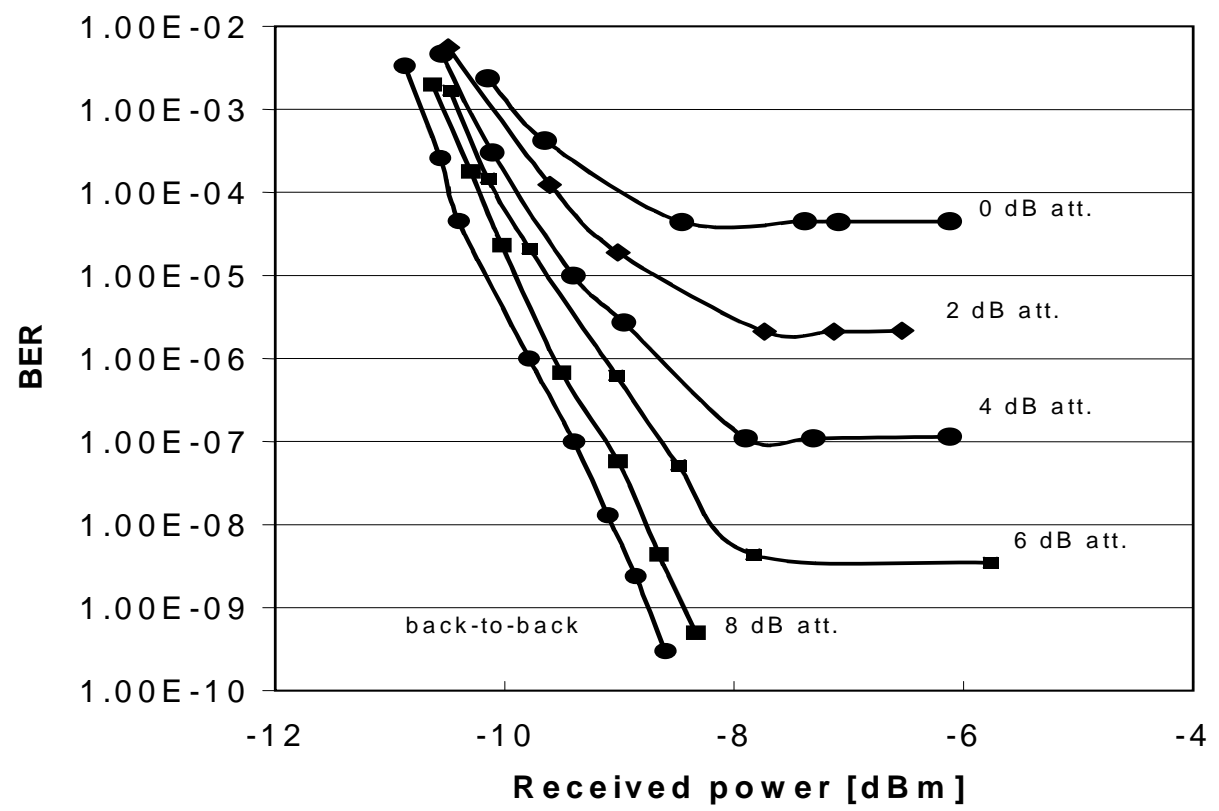

FIGURE 6 


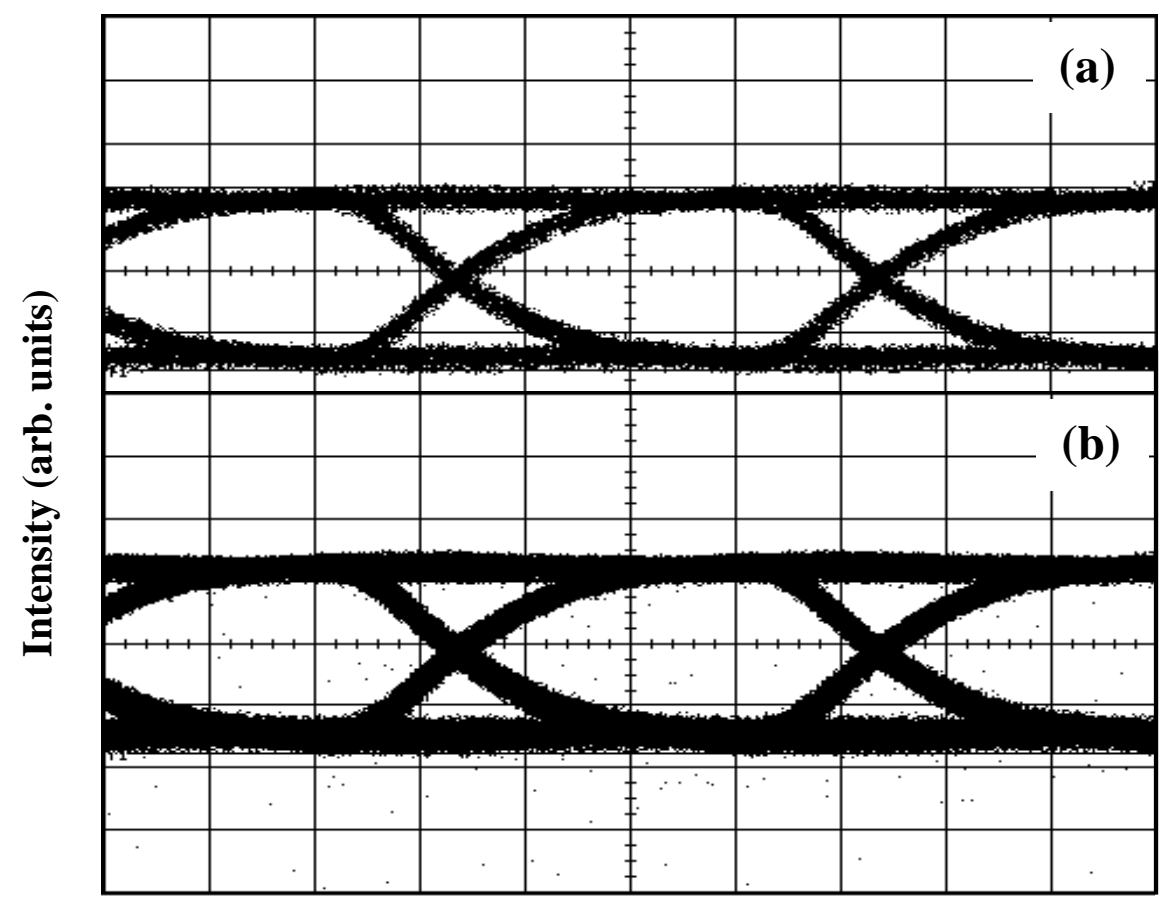

Time, 100 ps/div

\section{FIGURE 7}

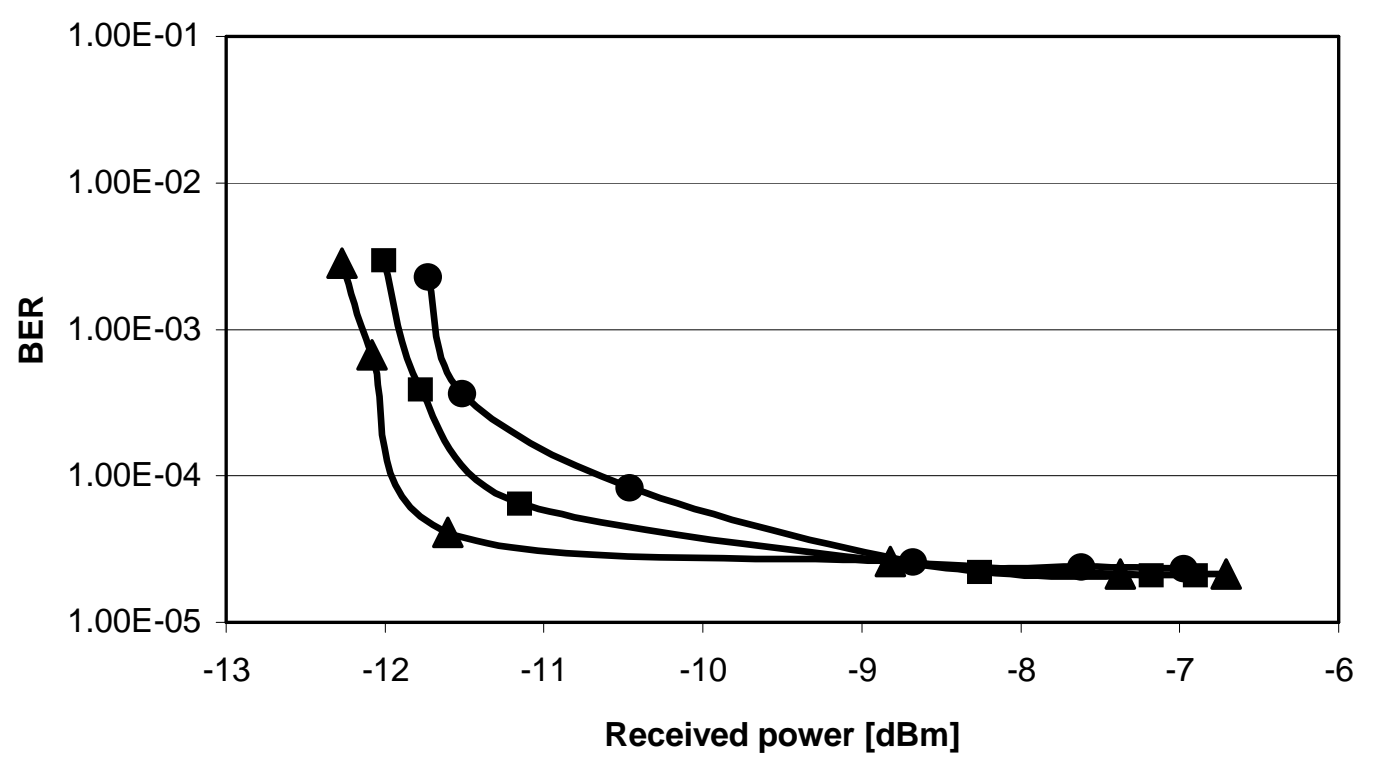

FIGURE 8 